\title{
Capacidad de reinicio de la meiosis de ovocitos provenientes de folículos de varios tamaños obtenidos de ovarios de camal
}

\section{Competence to restart meiosis of oocytes from different follicle sizes obtained from slaughterhouse ovaries}

\author{
Luis Ayala G. ${ }^{1,2}$, Tito Palacios C. ${ }^{2}$, Pedro Nieto E. ${ }^{1}$, Guido Calle O. ${ }^{1}$, Ramiro Rodas \\ C. ${ }^{1}$, Jorge Dutan S. ${ }^{1}$, Yury Murrillo ${ }^{1}$, Jorge Samaniego C. ${ }^{1}$
}

\section{Resumen}

Se evaluó la capacidad de los ovocitos de folículos según su tamaño para reiniciar la meiosis. Los folículos se midieron y clasificaron como Grupo 1 (G1 $<4 \mathrm{~mm}$ ), Grupo 2 (G2 4$8 \mathrm{~mm}$ ) y Grupo 3 (G3>8 mm). La aspiración se realizó por grupo con una aguja $21 \mathrm{G}$ conectada a una bomba de vacío, con una presión de $65 \mathrm{mmHg}$. Los complejos cúmulos de ovocitos (COC) recuperados se clasificaron como adecuados y no adecuados para la producción de embriones in vitro, de acuerdo con las características del cúmulo y del citoplasma. Los COC elegibles e inadecuados se valoraron con la prueba de azul brillante de Cresilo (BCB) y se clasificaron en $\mathrm{BCB}+\mathrm{y} \mathrm{BCB}$-. La maduración in vitro (IVM) se llevó a cabo en microgotas, incubadas en una cámara de $\mathrm{CO}_{2}$ al $5 \%, 38.5^{\circ} \mathrm{C}$ y $90 \%$ de humedad durante 24 horas. La progresión meiótica se determinó por extrusión del corpúsculo polar mediante epifluorescencia bajo un microscopio invertido. La morfometría de ovocitos se estableció mediante una cámara de alta definición (Excelis AU-600-HD) y un software (AmScope v.3.7). El porcentaje de recuperación de ovocitos fue mayor de 63\%. Los folículos de G2 proporcionaron un mayor porcentaje de COC elegibles (65.7\%), donde el $59 \%$ de este grupo se clasificó como $\mathrm{BCB}+$. Los ovocitos aptos de $\mathrm{G} 1$ y G2 reanudaron la meiosis en un $75 \%$. Además, se observó que los ovocitos después de IVM redujeron su diámetro. Se concluye que los ovocitos de folículos entre 4-8 $\mathrm{mm}(\mathrm{G} 2)$ proporcionan un mayor porcentaje de COC maduros; sin embargo, el 50\% de folículos $<4 \mathrm{~mm}$ (G1) son una fuente prometedora de ovocitos viables, por lo que deben usarse para la producción de embriones in vitro.

Palabras clave: ovocitos; azul brillante de cresilo; tamaño folicular; maduración in vitro

${ }^{1}$ Carrera de Medicina Veterinaria Zootecnia, Facultad de Ciencias Agropecuarias, Universidad de Cuenca, Ecuador

${ }^{2}$ Facultad de Ciencias Veterinarias, Instituto de Posgrados, Universidad Técnica de Manabi, Ecuador

${ }^{3}$ E-mail: luis.ayala@ucuenca.edu.ec

Recibido: 13 de noviembre de 2018

Aceptado para publicación: 4 de junio de 2019 
The competence of oocytes according to follicle size to restart meiosis was evaluated. The follicles were measured and classified as Group 1 (G1 <4 mm), Group 2 (G2 4-8 mm) and Group 3 (G3 $>8 \mathrm{~mm}$ ). The aspiration was performed by group with a $21 \mathrm{G}$ needle connected to a vacuum pump, with a pressure of $65 \mathrm{mmHg}$. The complex oocyte clusters (COCs) recovered were classified as suitable and unsuitable to produce embryos in vitro, according to the characteristics of the cumulus and the cytoplasm. Eligible and unsuitable COCs were assessed with the Brilliant Cresyl Blue (BCB) and were classified into $\mathrm{BCB}+$ and $\mathrm{BCB}$-. In vitro maturation (IVM) was carried out in microdroplets, incubated in a $5 \% \mathrm{CO}_{2}$ chamber, $38.5^{\circ} \mathrm{C}$ and $90 \%$ humidity for 24 hours. The meiotic progression was determined by extrusion of the polar corpuscle by epifluorescence under an inverted microscope. Oocyte morphometry was established using a high definition camera (Excelis AU-600-HD) and software (AmScope v.3.7). The recovery percentage of oocytes was greater than $63 \%$. The $\mathrm{G} 2$ follicles provided a higher percentage of eligible COCs $(65.7 \%)$, where $59 \%$ of this group was classified as $\mathrm{BCB}+$. The fit oocytes of $\mathrm{G} 1$ and $\mathrm{G} 2$ resumed meiosis by $75 \%$. In addition, it was observed that oocytes after IVM reduced their diameter. It is concluded that follicle oocytes between $4-8 \mathrm{~mm}(\mathrm{G} 2)$ provide a higher percentage of mature COCs; however, $50 \%$ of follicles $<4 \mathrm{~mm}(\mathrm{G} 1)$ are a promising source of viable oocytes, so they should be used for in vitro embryo production.

Key words: oocytes; brilliant cresyl blue; follicle size; in vitro maturation

\section{INTRODUCCIÓN}

El ovocito bovino adquiere competencia durante el desarrollo folicular inicial hasta la fecundación. Se ha descrito que esta competencia implica la remodelación de la cromatina en la vesícula germinal (GV), la cual en ovocitos en crecimiento no se encuentra condensada, pero conforme avanza la etapa de desarrollo experimenta un cambio dramático en la organización nuclear, permitiendo una condensación progresiva, formando una heterocromatina en estrecha aposición con el núcleo (Zuccotti et al., 1995; Albertini et al., 2003).

La capacidad de un ovocito para desarrollar un embrión depende de la cantidad de información específica en forma de ARNm o proteínas (Sirard, 2001). Así mismo, esta capacidad de desarrollo la adquiere gradualmente y aumenta concomitantemente con el tamaño folicular (Machatkova et al., 2004); por lo tanto, para mejorar el potencial de de- sarrollo in vitro, deben usarse ovocitos que hayan recibido instrucciones foliculares óptimas antes de su recolección (Gandolfi $\mathrm{T}$ y Gandolfi F, 2001).

En la producción de embriones in vitro se utilizan ovocitos provenientes de folículos entre 2 y $8 \mathrm{~mm}$, ya que autores como Motlik et al. (1984) establecieron que apenas una porción limitada de ovocitos con diámetro aproximado de $110 \mu \mathrm{m}$, provenientes de folículos de $0.5 \mathrm{~mm}$ reanudan la meiosis. Sin embargo, Arlotto et al. (1990) observaron que se producía reanudación de meiosis en ovocitos de 95-105 micras, de allí que la adquisición de competencia meiótica parece correlacionarse no solo con el tamaño del ovocito y del folículo, sino también con la morfología de las células del cúmulus y la actividad transcripcional (Motlik et al., 1984; Crozet et al., 1986).

En este contexto, De La Fuente y Eppig (2001) describieron que las células de la granulosa son reguladoras importantes de los 
eventos finales de diferenciación ovocitaria, ya que las células del cúmulus se comunican entre sí y con el ovocito desde las primeras etapas de crecimiento folicular, por medio de una extensa red de uniones GAP, que permite la transferencia bidireccional de pequeñas moléculas como nutrientes y ARNm entre células somáticas y germinales (De Loos et al., 1989); además, esta interacción es requerida para la actividad transcripcional progresiva de los ovocitos cultivados in vitro (De La Fuente y Eppig, 2001)

Estudios realizados en el bovino señalan que los complejos cúmulus ovocitos (COC) aislados de folículos antrales y seleccionados únicamente por criterios morfológicos para la producción de embriones in vitro, representan una población heterogénea caracterizada por diferentes grados funcionales de comunicación mediadas por uniones GAP. En la vaca en particular, solo el $60 \%$ de los COC aislados de folículos de 2-6 mm muestran un acoplamiento regular entre ovocitos y las células del cúmulus (Luciano et al., 2004). Esta forma rutinaria de valoración de la calidad de los COC entre otros factores no ha permitido mejorar los resultados finales de la producción de embriones in vitro (PIV) en los últimos 40 años (Jeong et al., 2009).

En los últimos años se han buscado alternativas que permitan seleccionar ovocitos competentes en forme rápida y económica, que permitan reducir la heterogeneidad de los COC obtenidos de ovarios de matadero (Karami y Mirshamsi, 2012). Una de estas utiliza el Azul Brillante de Cresilo (BCB) para evaluar la actividad de la glucosa-6-fosfato deshidrogenasa (G6PD), enzima sintetizada en la mitad de la primera fase (S) de crecimiento ovocitario y que disminuye conforme este termina su fase de crecimiento. La tinción $\mathrm{BCB}$ ha demostrado ser un método no invasivo y confiable para la selección de ovocitos bovinos (Pujol et al., 2004; Bhojwani et al., 2007), debido a que permite cuantificar la actividad de la G6PDH, tomando como principio a ovocitos que retienen la tinción como aquellos que terminaron su crecimien- to $(\mathrm{BCB}+)$ y como ovocitos incoloros a aquellos que se encuentran en crecimiento, por lo que son considerados BCB negativos (Ayala et al., 2018). Sin embargo, Karami et al. (2014) determinaron que la prueba del BCB por sí sola no es determinante para seleccionar ovocitos competentes para la producción de embriones in vitro.

Por lo antes expuesto, se ha planteado valorar la capacidad de reinicio de la meiosis que poseen los ovocitos provenientes de folículos de tamaños diferentes, seleccionados con la técnica tradicional (morfología de las células de cúmulus y el citoplasma), más el test del Azul Brillante de Cresilo (BCB) y su relación con la morfometría (diámetro) del ovocito.

\section{Materiales y Métodos}

Todos los materiales de plástico de cultivo utilizados en el estudio se obtuvieron de Falcon (USA), mientras que los productos químicos y medios se adquirieron de Sigma (USA). La investigación se realizó en el laboratorio de Biotecnología de la Universidad de Cuenca, Ecuador.

\section{Colección de Ovarios y Aspiración Folicular}

Los ovarios bovinos fueron obtenidos del matadero local (EMURPLAG) y transportados al laboratorio a $37^{\circ} \mathrm{C}$ en solución salina que contenía $50 \mathrm{UI} / \mathrm{ml}$ de penicilina y $50 \mu \mathrm{g} / \mathrm{ml}$ de estreptomicina. En el laboratorio se lavaron tres veces en solución salina atemperada y todo el tejido adyacente fue removido. Los folículos fueron medidos y categorizados en tres grupos (Grupo 1: $<4 \mathrm{~mm}$; Grupo 2: 4-8 mm; Grupo 3: $>8 \mathrm{~mm}$ ). Se hizo la punción de los folículos de cada grupo mediante una aguja de calibre $21 \mathrm{G}$ acoplada a una bomba de vacío (BV-003D ${ }^{\circledR}$, WTA) con una presión constante de $65 \mathrm{mmHg}$ y conducidos a un tubo Falcón de $50 \mathrm{ml}$, donde permanecieron por 15 min hasta la formación del sedimento. Con 
una pipeta Pasteur se colocó el sedimento en placas petri cuadriculadas de $95 \mathrm{~mm}^{2}$ para la búsqueda de COCs mediante estereomicroscopía.

\section{Clasificación Morfológica}

Se basó en las características morfológicas de las células del cúmulus y del citoplasma del ovocito siguiendo los criterios descritos por Hawk y Wall (1994), quienes los categorizaron en tipo A (ovocitos de apariencia compacta, con varias capas de células, granulosa adherida, citoplasma homogéneo, denso y finamente granulado ), tipo B (usualmente granulosa adherida, cúmulo compacto con pocas a varias capas, cubriendo al menos la mitad de la zona pelúcida, citoplasma irregular, denso y granulado) y tipo $\mathrm{C}$ (cúmulo parcial o completamente expandido y disperso, presencia de material extracelular, cúmulo descolorido, corona radiata desnuda, granulado grueso, mezcla de áreas muy claras o muy oscuras, citoplasma descolorido y deforme). Los COC fueron agrupados en aptos (categorías $\mathrm{A}+\mathrm{B}$ ) y no aptos (categoría C).

\section{Actividad Enzimática Glucosa-6-fosfato Deshidrogenasa (G6PD)}

Se realizó mediante la tinción vital del Azul Brillante de Cresilo (B-5388, Sigma). El 50\% de los COC de cada grupo se colocó en gotas de $50 \mu 1$ de BCB a una concentración de $26 \mu 1$, previamente diluida en PBS de Dulbecco, e introducidos a una cámara de maduración con atmósfera controlada por un tiempo de $90 \mathrm{~min}$. Luego, se lavaron las gametas en Hepes-Fluido Sintético Oviductal (H-SOF) y se evaluaron en estereomicroscopía con base a los criterios de: ovocitos con citoplasma teñido «positivos» $(\mathrm{BCB}+) \mathrm{y}$ ovocitos que no tiñeron el citoplasma «negativos» (BCB-) (Catala et al., 2011).

\section{Morfometría de Ovocitos}

Los ovocitos que fueron sometidos a la tinción de BCB se utilizaron para medir lamorfometría en la fase de pre-maduración (diámetro del ovocito, sin zona pelúcida).
Para la determinación pos-maduración se tomó el $25 \%$ de los COCs maduros. Fueron denudados con hialuronidasa a una concentración de $1 \mathrm{mg} / \mathrm{ml}(\mathrm{p} / \mathrm{v})$ por $2 \mathrm{~min}$. Para la valoración se utilizó el software AmScope v.3.7 calibrado para realizar mediciones en micras, el cual funciona con una cámara de alta definición (Excelis AU-600-HD) acoplada a un microscopio con un lente 100x.

\section{Maduración in vitro y Progresión Meiótica}

El $50 \%$ de los COC restantes fueron lavados en medio de maduración y colocados en seis microgotas. El medio de maduración consistió en TCM 199 suplementado con 10\% albumina sérica bovina (BSA) al 1\%, $100 \mu \mathrm{g} /$ $\mathrm{ml}$ de piruvato de sodio, $0.75 \mathrm{mg} / \mathrm{ml}$ de Lglutamina, $4 \mu \mathrm{g} / \mathrm{ml}$ de FSH-p (Folltropin ${ }^{\circledR}$, Bioniche, Canadá), $2 \mu \mathrm{g} / \mathrm{ml}$ de estradiol y 250 $\mu \mathrm{g} / \mathrm{ml}$ de gentamicina por $24 \mathrm{~h}$ en una cámara de maduración controlada al $5 \%$ de $\mathrm{CO}_{2}, 90 \%$ de humedad y $38.5^{\circ} \mathrm{C}$. Luego, los COC fueron observados bajo una lupa estereoscópica para verificar la expansión del cúmulo y el grado mitogénico.

Para la determinación de la progresión meiótica, los COC fueron denudados mediante hialuronidasa a una concentración de $1 \mathrm{mg} / \mathrm{ml}(\mathrm{p} / \mathrm{v})$ por $2 \mathrm{~min}$ y expuestos al fluorocromo Hoechst (33342, Sigma) en concentración de $1 \mathrm{ig} / \mathrm{ml}(\mathrm{p} / \mathrm{v})$ por $10 \mathrm{~min}$. La evaluación de la progresión meiótica se determinó por el estado de la vesícula germinal $(\mathrm{GV})$, considerando como no maduro cuando esta se encontró intacta, y como ovocito maduro cuando el núcleo se encontraba en metafase I (MI), anafase-telofase I (An-Tel I), metafase II (MII; primer corpúsculo polar). Los ovocitos fueron evaluados bajo un microscopio invertido fluorescente.

\section{Diseño Experimental}

El estudio incluyó tres tratamientos según el tamaño de los folículos (Grupo 1: $<4$ $\mathrm{mm}$; Grupo 2: 4-8 mm; Grupo 3: $>8 \mathrm{~mm}$ ) y 14 repeticiones. En primera instancia se relacionó 
el tamaño del folículo y el porcentaje de recuperación de los COC. Luego se determinó la calidad de los COC recuperados en cada tratamiento (grupo) basado en la morfológica de las células del cúmulus y el citoplasma (aptos y no aptos). En un tercer momento se valoró la actividad enzimática de la G6PD mediante el azul brillante de cresilo $(\mathrm{BCB}+\mathrm{y}$ BCB-), de los COC clasificados como aptos y no aptos. Finalmente, el estado de progresión meiótica y diámetro ovocitario fue relacionado con el tamaño del folículo.

El análisis estadístico se realizó mediante el programa SPSS v. 24. Se determinaron estadígrafos principales. Para establecer relación se aplicó la prueba de Chi cuadrado al $5 \%$. Para las variables cuantitativas se utilizó la prueba de análisis de varianza y para comparar medias se empleó la prueba de Tukey al 5\%.

\section{Resultados y Discusión}

Se aspiraron 3373 folículos y se recuperaron 2300 COC (68.18\%); de estos, 1270 pertenecían a G1, 817 a G2 y 213 a G3 $(p<0.05)$. Este comportamiento es similar al obtenido por Priscilla y Balakrishnan (2011), quienes obtuvieron un mayor número de COC al aspirar folículos $<4 \mathrm{~mm}$, seguidos por los de 4-8 mm. Por otro lado, se obtuvo el 76.2\% de recuperación en $\mathrm{G} 2$, el cual fue significativamente diferente de G1 (64.77\%) y de G3 $(63.4 \%)(p<0.05)$. La tasa de recuperación obtenida en G2 es superior a la reportada por Perea et al. (2017), quienes describen un $64.6 \%$ de recuperación ovocitaria al emplear una presión de vacío de $65 \mathrm{mmHg}$. Sin embargo, fue inferior a las tasas de Lonergan et al. (1994) de 77\% en folículos $>6 \mathrm{~mm}$ y de $87.7 \%$ al aspirar folículos de 2-6 $\mathrm{mm}$ (Cuadro 1).

El Cuadro 2 expone la relación entre el número de COC clasificados morfológicamente (células del cúmulus y citoplasma) como aptos o no aptos y el tamaño folicular. E1 grupo G2 presentó mayor porcentaje
$(65.7 \%)$ de COC aptos $(p<0.05)$. Si bien estos valores fueron superiores a los obtenidos por Pavlok et al. (1992), quienes alcanzaron $40.1 \%$ de ovocitos aptos al puncionar folículos de 4-8 $\mathrm{mm}$, la diferencia podría ser explicada por la presión de aspiración que utilizaron en su investigación; sin embargo, la tendencia a obtener mayor cantidad de COC de folículos 4-8 mm es similar. Así mismo, los valores obtenidos en este trabajo fueron menores al $89 \%$ de ovocitos aptos establecido por Hasler (1998), comportamiento que pudiera ser atribuido igualmente a la presión de aspiración $(50 \mathrm{mmHg})$ y al tipo de aguja utilizada $(17 \mathrm{G}$, $57 \mathrm{~cm}$ ). Estos comportamientos coinciden con el postulado propuesto por Wani et al. (2013), quienes determinan una relación entre el tamaño folicular y la competencia ovocitaria.

Por otra parte, al valorar el porcentaje de COC aptos y no aptos dentro de un mismo grupo experimental, se estableció que G2 permite recuperar casi el doble de COCs aptos con relación a los no aptos $(\mathrm{p}<0.05)$. En contraposición, el porcentaje de COC no aptos fue mayor en G1 $(p<0.05)$. Esto coincide con Lonergan et al. (1994), quienes determinaron que se puede obtener un mayor porcentaje de ovocitos viables de folículos $>6$ $\mathrm{mm}$. En forma similar, Yoon et al. (2000) obtuvieron ovocitos con más de tres capas de células del cúmulus y con citoplasma normal al aspirar folículos entre 3 y $8 \mathrm{~mm}$.

Mediante la prueba de BCB se determinó que un $59 \%$ de los COC clasificados como aptos del grupo $\mathrm{G} 2$ fueron $\mathrm{BCB}+$, siendo significativamente diferentes $(\mathrm{p}<0.05) \mathrm{de}$ G1 (44.6\%) y G3 (35.7\%) (Cuadro 3). Comportamiento similar fue obtenido por Karami et al. (2014) con folículos entre 3-6 mm $(64.5 \% \mathrm{BCB}+)$ y $>6 \mathrm{~mm}(59.3 \% \mathrm{BCB}+)$, factor que pude estar relacionado al grado de maduración citoplasmática, número de copias de ADN mitocondrial (Mohammadi et al., 2011) y diámetro y volumen citoplasmático (Pujol et al., 2004). Además, Opiela et al. (2010) señalaron que ovocitos BCB- tienen menores tasas de transcripción relacionado 
Cuadro 1. Número de folículos bovinos aspirados y media y porcentaje de COC recuperados de folículos de tres tamaños

\begin{tabular}{lccc}
\hline & G1 $(<4 \mathrm{~mm})$ & $\mathrm{G} 2(4-8 \mathrm{~mm})$ & $\mathrm{G} 3(>8 \mathrm{~mm})$ \\
\hline Folículos aspirados & $1964^{\mathrm{c}}$ & $1073^{\mathrm{b}}$ & $336^{\mathrm{a}}$ \\
COC recuperados & $1270^{\mathrm{c}}$ & $817^{\mathrm{b}}$ & $213^{\mathrm{a}}$ \\
Porcentaje de recuperación & $64.7^{\mathrm{a}}$ & $76.1^{\mathrm{b}}$ & $63.4^{\mathrm{a}}$ \\
\hline
\end{tabular}

${ }^{a, b}$ Valores con letras diferentes indican diferencia estadística (Chi cuadrado, $p<0.05$ )

Cuadro 2. Número y porcentaje de COC aptos y no aptos, según las características morfológicas de las células del cúmulus y del citoplasma del ovocito (Hawk y Wall, 1994), provenientes de folículos de tres tamaños

\begin{tabular}{lccc}
\hline COC & $\begin{array}{c}\mathrm{G},<4 \mathrm{~mm} \\
\mathrm{n}(\%)\end{array}$ & $\begin{array}{c}\mathrm{G} 2,4-8 \mathrm{~mm} \\
\mathrm{n}(\%)\end{array}$ & $\begin{array}{c}\mathrm{G} 3,>8 \mathrm{~mm} \\
\mathrm{n}(\%)\end{array}$ \\
\hline Aptos (A, B) & $539(42.4)^{\mathrm{aA}}$ & $537(65.7)^{\mathrm{cB}}$ & $117(54.9)^{\mathrm{bA}}$ \\
No aptos (C) & $731(57.6)^{\mathrm{cB}}$ & $280(34.3)^{\mathrm{aA}}$ & $96(45.1)^{\mathrm{bA}}$ \\
\hline
\end{tabular}

$\mathrm{a}, \mathrm{b}$ Letras diferentes dentro de columnas indican diferencia estadística (Tukey, $\mathrm{p}<0.05$ )

$A, B$ Letras diferentes dentro de filas indican diferencia estadística (Tukey, $p<0.05$ )

Cuadro 3. Número y porcentaje de COC recuperados de folículos de tres tamaños y su actividad al azul brillante de cresilo (BSB)

\begin{tabular}{lcccc}
\hline COC & Actividad & $\begin{array}{c}\mathrm{G} 1,<4 \mathrm{~mm} \\
\mathrm{n}(\%)\end{array}$ & $\begin{array}{c}\mathrm{G} 2,4-8 \mathrm{~mm} \\
\mathrm{n}(\%)\end{array}$ & $\begin{array}{c}\mathrm{G} 3,>8 \mathrm{~mm} \\
\mathrm{n}(\%)\end{array}$ \\
\hline Aptos $(\mathrm{A}, \mathrm{B})$ & $\mathrm{BCB}+$ & $112(44.6)^{\mathrm{aA}}$ & $138(59.0)^{\mathrm{aB}}$ & $20(35.7)^{\mathrm{aA}}$ \\
& BCB- & $139(55.4)^{\mathrm{aB}}$ & $96(41.0)^{\mathrm{aA}}$ & $36(64.3)^{\mathrm{aB}}$ \\
No aptos (C) & BCB+ & $143(40.2)^{\mathrm{aA}}$ & $65(50.4)^{\mathrm{aA}}$ & $18(40.9)^{\mathrm{aA}}$ \\
& BCB- & $213(59.8)^{\mathrm{aA}}$ & $64(49.6)^{\mathrm{aA}}$ & $26(59.1)^{\mathrm{aA}}$ \\
\hline
\end{tabular}

$a, b$ Letras diferentes dentro de filas indican diferencia estadística (Tukey, $p<0.05$ )

$A, B$ Letras diferentes dentro de columnas indican diferencia estadística (Tukey, $p<0.05$ )

a una baja biosíntesis mitocondrial, concluyendo que esta es la razón principal de la baja competencia ovocitaria en este tipo de COC.

Al analizar la actividad de los COC clasificados como no aptos no se observó diferencia significativa entre grupos (Cuadro 3), pero se determinó que alrededor del $44 \%$ habían culminado su crecimiento (BCB+). Conducta similar fue observada por Ayala et al. (2018) al analizar ovocitos clasificados como $\mathrm{C}$ y D, determinado que alrededor del $60 \%$ de estas estructuras eran $\mathrm{BCB}+$, indicando que ese resultado se debe a factores técnicos (longitud, bisel y diámetro de aguja; así como a la presión de aspiración) que pro- 
Cuadro 4. Ovocitos aptos y no aptos, según las características morfológicas de las células del cúmulus y del citoplasma del ovocito (Hawk y Wall, 1994), provenientes de folículos de tres tamaños que llegaron a completar la fase de maduración (media \pm error estándar y porcentaje

\begin{tabular}{lccc}
\hline Ovocitos & G1 $(<4 \mathrm{~mm})$ & $\mathrm{G} 2(4-8 \mathrm{~mm})$ & $\mathrm{G} 3(>8 \mathrm{~mm})$ \\
\hline Aptos & & & \\
$\quad$ Maduros & $13.9 \pm 1.41^{\mathrm{b}}(74.9)^{\mathrm{B}}$ & $13.5 \pm 1.78^{\mathrm{b}}(75.6)^{\mathrm{B}}$ & $2.6 \pm 0.35^{\mathrm{a}}(61.7)^{\mathrm{A}}$ \\
$\quad$ Inmaduros & $4.6 \pm 0.55^{\mathrm{b}}(25.1)^{\mathrm{A}}$ & $4.4 \pm 0.87^{\mathrm{b}}(24.4)^{\mathrm{A}}$ & $1.6 \pm 0.46^{\mathrm{a}}(38.3)^{\mathrm{A}}$ \\
& & \\
No aptos & & \\
$\quad$ Maduros & $13.7 \pm 1.96^{\mathrm{b}}(59.6)^{\mathrm{B}}$ & $5.5 \pm 0.93^{\mathrm{a}}(60.6)^{\mathrm{B}}$ & $1.4 \pm 0.31^{\mathrm{a}}(44.2)^{\mathrm{A}}$ \\
$\quad$ Inmaduros & $9.3 \pm 0.69^{\mathrm{b}}(40.4)^{\mathrm{A}}$ & $3.6 \pm 0.53^{\mathrm{a}}(39.4)^{\mathrm{A}}$ & $1.7 \pm 0.30^{\mathrm{a}}(55.8)^{\mathrm{A}}$ \\
\hline a,b Letras diferentes dentro de filas indican diferencia estadística (Tukey, p<0.05) \\
A,B Letras diferentes dentro de columnas indican diferencia estadística (Tukey, $<<0.05)$
\end{tabular}

Cuadro 5. Diámetro de ovocitos clasificados como aptos y no aptos, pre y post maduración provenientes de folículos de tres tamaños, valorando el citoplasma (media \pm error estándar)

\begin{tabular}{lccc}
\hline Citoplasma & G1 $(<4 \mathrm{~mm})$ & G2 $(4-8 \mathrm{~mm})$ & G3 $(>8 \mathrm{~mm})$ \\
\hline Aptos & & & \\
Pre-maduración $(\mu \mathrm{m})$ & $121.1 \pm 0.41^{\mathrm{a}}$ & $121.9 \pm 0.42^{\mathrm{ab}}$ & $124.0 \pm 1.13^{\mathrm{b}}$ \\
Pos-maduración $(\mu \mathrm{m})$ & $117.7 \pm 0.43^{\mathrm{a}}$ & $120.1 \pm 0.52^{\mathrm{b}}$ & $120.3 \pm 0.94^{\mathrm{b}}$ \\
Diferencia $(\mu \mathrm{m})$ & $-3.4 \pm 0.62^{\mathrm{b}}$ & $-1.9 \pm 0.69^{\mathrm{a}}$ & $-3.7 \pm 1.20^{\mathrm{b}}$ \\
No aptos & & & \\
Pre-maduración $(\mu \mathrm{m})$ & $123.3 \pm 0.41^{\mathrm{a}}$ & $120.7 \pm 1.16^{\mathrm{a}}$ & $122.1 \pm 1.76^{\mathrm{a}}$ \\
Pos-maduración $(\mu \mathrm{m})$ & $115.9 \pm 0.43^{\mathrm{a}}$ & $120.1 \pm 0.52^{\mathrm{b}}$ & $120.3 \pm 0.94^{\mathrm{b}}$ \\
Diferencia $(\mu \mathrm{m})$ & $-7.3 \pm 0.74^{\mathrm{a}}$ & $-0.6 \pm 1.38^{\mathrm{b}}$ & $-1.8 \pm 1.95^{\mathrm{b}}$ \\
\hline
\end{tabular}

$a, b$ Letras diferentes dentro de filas indican diferencia estadística (Tukey, $p<0.05$ )

dujeron pérdida de las células del cúmulo (ovocitos tipo C); así mismo, los ovocitos tipo D debido a su madurez fisiológica fueron excluidos de la producción de embriones in vitro (Ireland et al., 2007; Ding et al., 2008).

Aproximadamente el $75 \%$ de ovocitos clasificados como aptos se obtuvieron en los grupos G1 y G2, siendo significativamentemenores en G3 (Cuadro 4). Además, un $60 \%$ de ovocitos clasificados como no aptos de los grupos G1 y G2 presentaron maduración, siendo una fuente interesante de gametas viables. Autores como Yoon et al. (2000) obtuvieron mayor tasa de maduración nuclear (metafase II) con folículos de 3-8 mm $(91 \%)$ y un $58 \%$ con folículos pequeños $(<3$ $\mathrm{mm}$ ), tendencia similar al encontrado en la presente investigación. Por otra parte, De Smedt et al. (1994), Crozet et al. (1995) y Wani et al. (2013) concluyeron que la adquisición de competencia meiótica ocurre progresivamente durante el crecimiento folicular, comportamiento semejante al de este estudio 
con los grupos G1 y G2; sin embargo, G3 mostró porcentajes inferiores a los descritos por Karami et al. (2015) quienes obtuvieron 81 y $90 \%$ de expansión de las células del cúmulus de folículos medianos $(6-9 \mathrm{~mm})$ y grandes (10-20 mm), respectivamente, diferencia que pudiera ser explicada por las diferentes técnicas de evaluación meiótica utilizadas para valorar la maduración.

Varios investigadores describen que los ovocitos adquieren competencia meiótica cuando alcanzan diámetros superiores a $110 \mu \mathrm{m}$, lo cual corresponde a folículos de 2-3 $\mathrm{mm}$ de diámetro (Ayala et al., 2018). Este concepto sugiere que los ovocitos de menor tamaño no han terminado la síntesis de ARN y, por consiguiente, se encuentran en fase de crecimiento; es decir, no han completado la maduración nuclear y citoplasmática. No obstante, la mayoría de estos se desarrollan hasta metafase II cuando el diámetro ovocitario supera los $110 \mu \mathrm{m}$ (Crozet et al., 1986).

En la valoración morfométrica de los ovocitos clasificados como aptos (sin considerar zona pelúcida) antes del proceso de maduración (Cuadro 5), se determinó que el diámetro fluctuaba entre 121 y $124 \mu \mathrm{m}$, siendo más grandes los de G3 $(124.0 \pm 1.13 \mu \mathrm{m})$ en comparación con los de G1 $(121.1 \pm 0.41 \mu \mathrm{m})$ $(\mathrm{p}<0.05)$. Estos valores son similares a los descritos por Arlotto et al. (1996), quienes determinan que los ovocitos provenientes de folículos 3-6 mm miden $123.5 \pm 8.1 \mu \mathrm{m}$ de diámetro, así como con De Wit y Kruip (2001), quienes describen ovocitos de 122.6 ìm. Por otro lado, Segura et al. (2015) indicaron que el diámetro del ovocito sin considerar zona pelúcida se encuentra entre 113.0 y $115 \mu \mathrm{m}$, aunque con una alta variabilidad.

Luego del proceso de maduración in vitro de los ovocitos clasificados como aptos, se observó que el diámetro del citoplasma disminuye en mayor grado en los grupos G1 $(3.4 \pm 0.62 \mu \mathrm{m})$ y G3 $(3.7 \pm 1.20 \mu \mathrm{m})$; $\sin$ embargo, en G2 si bien presentan el mismo patrón de comportamiento, la reducción del diámetro fue menor $(1.9 \pm 0.69 \mu \mathrm{m})$. En cuanto al diámetro de ovocitos no aptos pre y posmaduración, no se encontró diferencia significativa entre grupos.

\section{Conclusiones}

Los ovocitos provenientes de folículos entre 4-8 $\mathrm{mm}(\mathrm{G} 2)$ proporcionan mayor porcentaje de COCs maduros; sin embargo, el $50 \%$ de los folículos $<4 \mathrm{~mm}$ (G1) son una fuente promisoria de ovocitos viables, que llegan a completar el proceso de maduración nuclear, por lo cual deberían ser utilizados para la producción de embriones in vitro.

\section{Agradecimientos}

Un agradecimiento especial a la Dirección de Investigación de la Universidad de Cuenca (DIUC) por el aporte económico para la realización del estudio.

\section{Literatura Citada}

1. Albertini DF, Sanfins A, Combelles CM. 2003. Origins and manifestations of oocyte maturation competencies. Reprod Biomed Online 6: 410-415. doi: 10.1016/S1472-6483(10)62159-1

2. Arlotto TM, Leifried-Rutledge ML, First NL. 1990. Size distribution and meiotic competence of bovine primary oocytes from two locations in the ovary. Theriogenology 33: 188. doi: 10.1016/ 0093-691x(90)90612-w

3. Arlotto T, Schwartz JL, First NL, Leibfried-Rutledge ML. 1996. Aspects of follicle and oocyte stage that affect in vitro maturation and development of bovine oocytes. Theriogenology 45: 943956. doi: 10.1016/0093-691X(96)00024-6

4. Ayala L, Samaniego J, Nieto P, Rodas $R$, Dutan J, Calle G, Murillo $Y$, et al. 2018. Competencia del ovocito bovino obtenido por ovum pick-up valorado mediante el azul brillante de cresilo. Rev Inv Vet Perú 29: 552-558. doi: 10.15381/ rivep.v29i2.13816 
5. Bhojwani S, Alm H, Torner H, Kanitz $W$, Poehland R. 2007. Selection of developmentally competent oocytes through brilliant cresyl blue stain enhances blastocyst development rate after bovine nuclear transfer. Theriogenology 67: 341-345. doi: 10.1016/ j.theriogenology.2006.08.006

6. Catalá MG, Izquierdo D, Uzbekova S, Morató R, Roura M, Romaguera $R$, Papillier P, et al. 2011. Brilliant Cresyl Blue stain selects largest oocytes with highest mitochondrial activity, maturation-promoting factor activity and embryo developmental competence in prepubertal sheep. Reproduction 142: 517-527. doi: 10.1530/REP-10-0528

7. Crozet N, Kanka J, Motlik J, Fulka J. 1986. Nucleolar fine structure and RNA synthesis in bovine oocytes from antral follicles. Gamete Res 14: 65-73. doi: 10.1002/mrd.1120140108

8. Crozet N, Ahmed-Ali M, Dubos MP. 1995. Developmental competence of goat oocytes from follicles of different size categories following maturation, fertilization and culture in vitro. J Reprod Fertil 103: 293-298. doi: 10.1530/ jrf.0.1030293

9. De La Fuente R, Eppig JJ. 2001. Transcriptional activity of the mouse oocyte genome: companion granulosa cells modulate transcription and chromatin remodeling. Dev Biol 229: 224-236. doi: 10.1006/dbio.2000.9947

10. de Loos F, van Vliet C, van Maurik P, Kruip TA. 1989. Morphology of immature bovine oocytes. Gamete Res 24: 197-204. doi: 10.1002/mrd.1120240207

11. de Smedt V, Crozet N, Gall L. 1994. Morphological and functional changes accompanying the acquisition of meiotic competence in ovarian goat oocyte. J Exp Zool 269: 128-139. doi: 10.1002/ jez.1402690206

12. De Wit AA, Kruip TA. 2001. Bovine cumulus-oocyte-complex-quality is reûected in sensitivity for alpha-amanitin, oocyte-diameter and developmental capacity. Anim Reprod Sci 65: 51-65. doi: 10.1016/S0378-4320(00)00215-3

13. Ding LJ, Tian HB, Wang JJ, Chen J, Sha HY, Chen JQ, Cheng GX. 2008. Different intervals of ovum pick-up affect the competence of oocytes to support the preimplantation development of cloned bovine embryos. Mol Reprod Dev 75: 1710-1715. doi: 10.1002/ mrd.20922

14. Gandolfi TA, Gandolfi F. 2001. The maternal legacy to the embryo: cytoplasmic components and their effects on early development. Theriogenology 55: 1255-1276. doi: 10.1016/S0093-691X(01)-00481-2

15. Hasler JF. 1998. The current status of oocyte recovery, in vitro embryo production, and embryo transfer in domestic animals, with an emphasis on the bovine. J Anim Sci 76: 52-74. doi: 10.2527/1998.76suppl_352x

16. Hawk HW, Wall RJ. 1994. Improved yields of bovine blastocysts from in vitroproduced oocytes. I. Selection of oocytes and zygotes. Theriogenology 41: 1571-1583. doi: 10.1016/0093-691X(94)90822-Z

17. Ireland JJ, Ward F, Jimenez-Krassel F, Ireland JL, Smith GW, Lonergan $P$, Evans AC. 2007. Follicle numbers are highly repeatable within individual animals but are inversely correlated with FSH concentrations and the proportion of good-quality embryos after ovarian stimulation in cattle. Hum Reprod 22: 1687-1695. doi: 10.1093/humrep/dem071

18. Jeong WJ, Cho SJ, Lee HS, Deb GK, Lee YS, Kwon TH, Kong IK. 2009. Effect of cytoplasmic lipid content on in vitro developmental efficiency of bovine IVP embryos. Theriogenology 72:584-589. doi: 10.1016/j.theriogenology.2009.-04.015

19. Karami-Shabankareh H, Mirshamsi SM. 2012. Selection of developmentally competent sheep oocytes using the brilliant cresyl blue test and the relationship to follicle size and oocyte diameter. Small Ruminant Res 105: 244249. doi: 10.1016/J.SMALLRUMRES.2012.02.017 
20. Karami-Shabankareh H, Azimi G, Torki M. 2014. Developmental competence of bovine oocytes selected based on follicle size and using the brilliant cresyl blue (BCB) test. Iran J Reprod Med 12: 771-778.

21. Karami-Shabankareh H, Shahsavari MH, Hajarian H, Moghaddam G. 2015. In vitro developmental competence of bovine oocytes: effect of corpus luteum and follicle size. Iran J Reprod Med 13: 615-622.

22. Lonergan P, Monaghan P, Rizos D, Boland MP, Gordon I. 1994. Effect of follicle size on bovine oocyte quality and developmental competence following maturation, fertilization, and culture in vitro. Mol Reprod Dev 37: 48-53. doi: 10.1002/mrd.1080370107

23. Luciano AM, Modina S, Vassena $R$, Milanesi E, Lauria A, Gandolfi F. 2004. Role of intracellular cyclic adenosine 32, 52-monophosphate concentration and oocyte-cumulus cells communications on the acquisition of the developmental competence during in vitro maturation of bovine oocyte1. Biol Reprod 70: 465-472. doi: 10.1095/ biolreprod.103.020644

24. Machatkova M, Krausova K, Jokesova E, Tomanek M. 2004. Developmental competence of bovine oocytes: effects of follicle size and the phase of follicular wave on in vitro embryo production. Theriogenology 61: 329-335. doi: 10.1016/S0093-691X(03)00216-4

25. Mohammadi-Sangcheshmeh A, Held E, Ghanem N, Rings $F$, SalilewWondim D, Tesfaye D, Sieme H, et al. 2011. G6PDH-activity in equine oocytes correlates with morphology, expression of candidate genes for viability, and preimplantative in vitro development. Theriogenology 76: 1215-1226. https:// doi.org/10.1016/j.theriogenology.2011.05.025

26. Motlik J, Crozet N, Fulka J. 1984. Meiotic competence in vitro of pig oocytes isolated from early antral follicles. J Reprod Fertil 72: 323-328. doi: 10.1530/jrf.0.0720323

27. Opiela J, Lipiñski D, S $S^{3}$ mski R, Katska-Ksiazkiewicz L. 2010. Transcript expression of mitochondria related genes is correlated with bovine oocyte selection by BCB test. Anim Reprod Sci 118: 188-193. doi: 10.1016/ j.anireprosci.2009.07.007

28. Pavlok A, Lucas-Hahn A, Niemann H. 1992. Fertilization and developmental competence of bovine oocytes derived from different categories of antral follicles. Mol Reprod Dev 31: 63-67. doi: 10.1002/mrd.1080310111

29. Perea F, Quezada A, Mocha A, Argudo G, Ayala L, Mendez S, Soria M, et al. 2017. Efecto de la presión de vacío sobre las características funcionales de ovocitos bovinos obtenidos de ovarios de matadero. Maskana 8: 69-71.

30. Priscilla KL, Balakrishnan PP. 2011. Effect of follicle size on number and quality of oocytes in bovines. Indian $\mathrm{J}$ Anim Reprod 32: 55-56.

31. Pujol M, López-Béjar M, Paramio MT. 2004. Developmental competence of heifer oocytes selected using the brilliant cresyl blue (BCB) test. Theriogenology 61: 735-744. doi: 10.1016/S0093691X(03)00250-4

32. Segura G, Cortez J, Cayo I. 2015. Capacidad de maduración in vitro de ovocitos obtenidos de folículos de tres tamaños diferentes en bovinos. Spermova 5: 106-109.

33. Sirard MA. 2001. Resumption of meiosis: mechanism involved in meiotic progression and its relation with developmental competence. Theriogenology 55 1241-1254. doi: 10.1016/S0093691X(01)00480-0

34. Wani AR, Khan MZ, Sofi KA, Malik AA, Lone FA, Bhat FA. 2013. Effect of follicular size on in vitro maturation, fertilization and culture of sheep embryos. Iran J Vet Res 14: 299-304.

35. Yoon KW, Shin TY, Park JI, Roh S, Lim JM, Lee BC, Hwang WS, Lee ES. 2000. Development of porcine oocytes 
from preovulatory follicles of different sizes after maturation in media supplemented with follicular fluids. Reprod Fert Develop 12: 133-139. doi: 10.1071/RD00027
36. Zuccotti M, Piccinelli A, Giorgi Rossi P, Garagna S, Redi CA. 1995. Chromatin organization during mouse oocyte growth. Mol Reprod Dev 41: 479485. doi: $10.1002 / \mathrm{mrd} .1080410410$ 